\title{
Observation of backflow in the switch-on dynamics of a hybrid aligned nematic
}

\author{
S. A. Jewell ${ }^{\text {a) }}$ and J. R. Sambles \\ Thin Film Photonics Group, School of Physics, University of Exeter, Stocker Road, Exeter, EX4 4QL, \\ United Kingdom
}

(Received 17 September 2003; accepted 11 November 2003)

\begin{abstract}
The optical convergent-beam technique is used to measure, in $0.3 \mathrm{~ms}$ steps, the response of the director in a 4.6- $\mu \mathrm{m}$-thick ZLI-2293 filled hybrid aligned nematic cell when a $10 \mathrm{kHz}, 7 \mathrm{~V}_{\mathrm{rms}}$ ac voltage is applied to the cell. The total time taken for the reorientation process is $2.4 \mathrm{~ms}$, with backflow observed during the first $1.5 \mathrm{~ms}$ after the application of the voltage. The measured director profiles show excellent agreement with theoretical profiles produced from the Leslie-EriksenParodi theory using typical values for the viscosity coefficients. Fluid velocity profiles within the cell are also modeled. (C) 2004 American Institute of Physics. [DOI: 10.1063/1.1638903]
\end{abstract}

The thresholdless response of the hybrid aligned nematic (HAN) cell geometry to applied fields makes it an ideal structure for studying the physical properties of nematic liquid crystals. The HAN structure is composed of homogeneous alignment (the tilt angle, $\theta \approx 0^{\circ}$ ) on one surface, and homeotropic $\left(\theta=90^{\circ}\right)$ on the other. For a nonchiral material, this results in the director tilting through $90^{\circ}$ between the two substrates, with no twist. For a material of positive dielectric anisotropy and of nonequal splay and bend elastic constants, when no voltage is applied the variation in tilt angle through the cell is close to linear (a linear variation requires $K_{11}=K_{33}$ ). When a high voltage is applied the director becomes predominantly homeotropically aligned. The dynamic reorientation of the director as it switches from the $0 \mathrm{~V}$ to the high voltage state is very fast $(\approx \mathrm{ms})$ and can be modeled using the Leslie-Eriksen-Parodi (LEP) theory. ${ }^{1-4}$ This theory enables the dynamics of an anisotropic fluid to be determined by considering not only the flow, as in the isotropic case, but also the rotation and alignment of the material. In the case of the response of a one-dimensional HAN structure, the process is described by four Miesowicz coefficients $\left(\eta_{1}, \eta_{2}, \eta_{12}\right.$, and $\left.\gamma_{1}\right)$ and the tilt profile at each point in time can be determined by solving the LEP equations numerically. The results show that the switch-on reorientation process for $7 \mathrm{~V}_{\mathrm{rms}}$ ac applied across a $5 \mu \mathrm{m}$ HAN cell is around $2.5 \mathrm{~ms}$, which is considerably faster than that of an equal thickness $90^{\circ}$ twisted nematic cell. The form of the director during the dynamic process also suggests that backflow occurs during the initial $1.5 \mathrm{~ms}$, due to a coupling between shear and rotational motion in adjacent regions of the cell. ${ }^{5}$

The use of optical waveguides to study the static director profile in liquid crystal cells is well established. ${ }^{6-8}$ By collecting optical-intensity versus angle-of-incidence data for reflected and transmitted TM or TE polarized light incident on a cell, and comparing it to a multilayer optics model, detailed information about the director profile can be obtained. The convergent beam technique has recently been developed to allow this optical probing method to be used to study the dynamic properties of liquid crystal cells. ${ }^{9,10}$ In this

${ }^{\text {a)} E l e c t r o n i c ~ m a i l: ~ s . a . j e w e l l @ e x e t e r . a c . u k ~}$ case, an expanded beam from a $\mathrm{He}-\mathrm{Ne}$ laser is focused onto the cell, allowing optical waveguide modes to be excited over a range of angles of incidence simultaneously. The intensity of the reflected and transmitted light is then collected by a charge-coupled-device (CCD) array with a capture time in the submillisecond range, allowing the variation of optical intensity with time to be recorded during the director reorientation process in the liquid crystal cell. The incident light is either $p$ (transverse magnetic) or $s$ (transverse electric) polarized, and so eight datasets in total can be collected ( $p$ to- $p$ and $s$-to- $s$ conserving and $p$-to-s and $s$-to- $p$ converting for both transmission and reflection). Comparing these data with theory (as in the static case) for each time step allows the director profile at each point in time to be determined.

A 4.6 $\mu \mathrm{m}$ ZLI-2293 (Merck) filled HAN cell was constructed from indium-tin-oxide (ITO) coated glass substrates. A $30 \mathrm{~nm}$ layer of obliquely evaporated silicon monoxide was used as the homogeneous alignment layer, and a monolayer of octadecyltrimethoxysilane provided homeotropic alignment. The cell was index matched between two lowindex glass hemispheres and mounted at the center of the optical convergent-beam arrangement, as shown in Fig. 1.

A $7 \mathrm{~V}_{\mathrm{rms}}, 10 \mathrm{kHz}$ ac voltage was applied to the cell, and optical intensity versus angle-of-incidence data were collected as a function of time for transmitted and reflected signals as the director responded to the change in voltage.

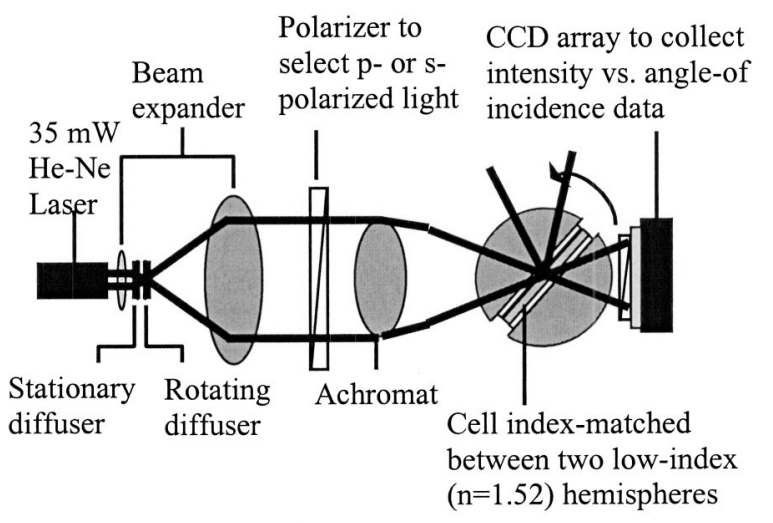

FIG. 1. Schematic diagram of the layout of the optical components used in the convergent-beam technique. 

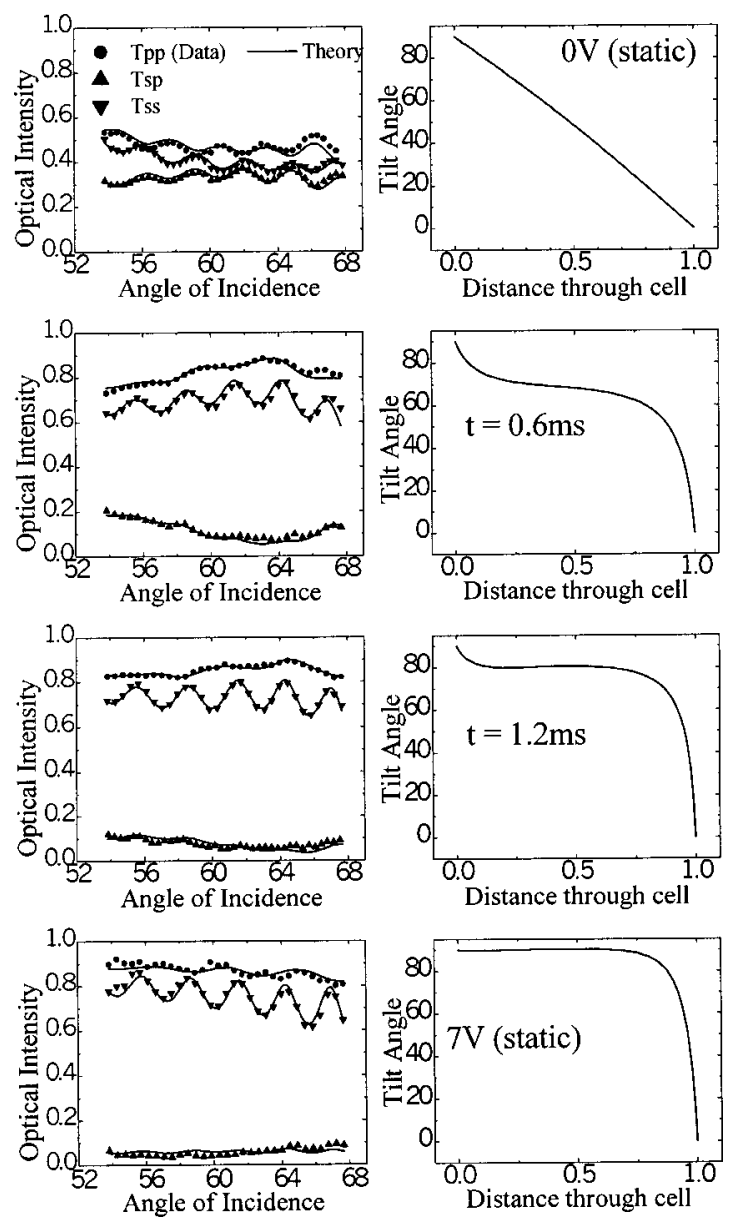

FIG. 2. Comparison between measured optical-intensity vs angle-ofincidence data (symbols) and theoretical data (lines) produced using a multilayer optics model, and the corresponding tilt angle (in degrees) vs fractional distance through the cell for a selection of times after the application of $7 \mathrm{~V}_{\mathrm{rms}}$ to the HAN cell.

The optical data showed that no further reorientation of the cell was distinguishable from the noise of the data after a reorientation time of around $2.4 \mathrm{~ms}$, with a particularly rapid change in the optical data, and hence the director profile, occurring over the first $1.5 \mathrm{~ms}$ after the voltage was applied.

To obtain the dynamic director profile at a particular time step, the transmitted and reflected optical intensity versus angle-of-incidence datasets were simultaneously fitted to a multilayer optics model, based on a $4 \times 4$ Berreman matrix procedure. ${ }^{11}$ The optimum result was determined by using a least-squares fitting procedure. The optical permittivity, absorption, and thickness of the ITO, silicon monoxide, and liquid crystal layers of the cell were used as fitting parameters along with the shape of the director profile. Examples of the fitted data and the corresponding director profiles are shown in Fig. 2. To allow for the rapid variation in optical intensity over the $0.3 \mathrm{~ms}$ exposure time of the CCD array, the time at which the first line of data was collected was approximated as $0.15( \pm 0.15) \mathrm{ms}$, with the subsequent lines of data defined in $0.3 \mathrm{~ms}$ intervals after that. These measured tilt profiles show good agreement with model profiles produced using the LEP theory, as illustrated in Fig. 3. In this case, the viscosity coefficients used to model the switch-on process were: $\eta_{1}=160 \pm 5 \mathrm{mPas}, \quad \eta_{2}=10 \pm 5 \mathrm{mPas}, \quad \eta_{12}=-100$ $\pm 50 \mathrm{mPas}$, and $\gamma_{1}=155 \pm 5 \mathrm{mPa} \mathrm{s}$, which are in agreement Downloaded 27 May 2008 to 144.173.6.22. Redistribution subject

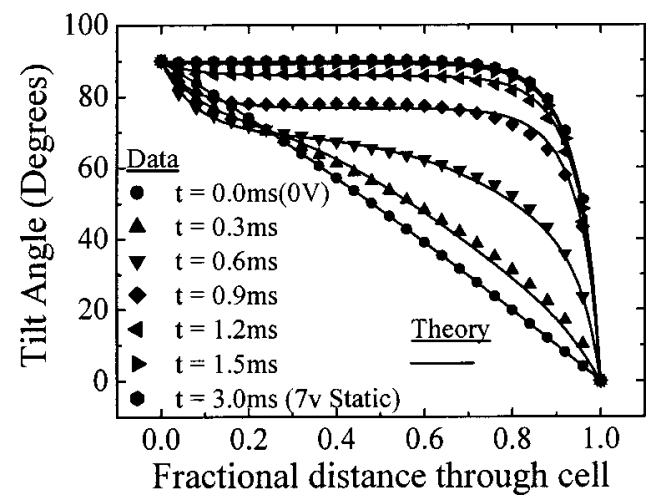

FIG. 3. Comparison between measured (symbols) HAN cell switch-on dynamic tilt profiles and modeled profiles (lines) produced using the LEP theory in conjunction with the viscosity coefficients given in the literature.

with typical values suggested by the manufacturer.

During the initial $1.5 \mathrm{~ms}$ reorientation process of the cell, backflow can be inferred as occurring close to the homeotropic surface of the cell. In this situation, the director becomes increasingly homogeneous in alignment, before reversing in direction and becoming homeotropically aligned. This process is driven by a coupling between rotational and shear flow motion in adjacent regions of the cell. This is reflected in the modeled fluid velocity profiles shown in Fig. 4, again produced using the LEP theory. During the first 0.5 $\mathrm{ms}$, there are two regions of high fluid velocity, with the direction of flow in opposing directions. It is the torque produced by the interaction of these two regions of mass fluid flow that results in the rotation of the director, and is referred to as backflow.

Further analysis of the director reorientation during the switch-on process shows that only two of the viscosity coefficients had any significant effect on the dynamic reorientation, with $\eta_{1}$ determining the degree of backflow and $\gamma_{1}$ governing the overall relaxation time. However, if shear flow is neglected altogether in the model (i.e., only the rotation of the director is considered) backflow does not occur, and the response time of the cell is considerably longer.

In conclusion, the variation in director profile with time when a $7 \mathrm{~V}_{\text {rms }}$ ac voltage was applied to a HAN cell has been measured using the convergent beam technique. The director was studied on a $0.3 \mathrm{~ms}$ time scale, and backflow observed

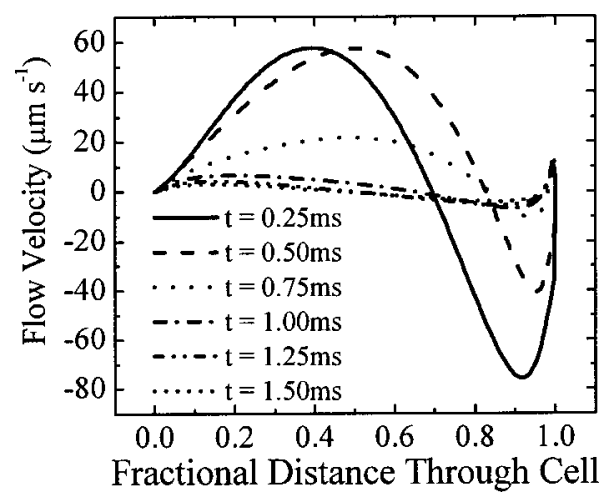

FIG. 4. Modeled flow velocity profiles (in $0.25 \mathrm{~ms}$ steps) for the first $1.5 \mathrm{~ms}$ of the switch-on process of a HAN cell, generated using the LEP theory and viscosity coefficients given in the literature. 
during the initial $1.5 \mathrm{~ms}$ of the $2.4 \mathrm{~ms}$ reorientation process. Measured director profiles showed excellent agreement with model profiles produced using the LEP theory, and the variation in flow velocity at points through the cell with time has also been modeled. Finally, further modeling suggests that the overall response time for the cell is governed by the rotational viscosity coefficient $\gamma_{1}$ and the degree of backflow is determined by the shear viscosity coefficient $\eta_{1}$.

The authors wish to thank EPSRC and Hewlett-Packard Laboratories, Bristol, for the financial support of this work.
${ }^{1}$ J. L. Eriksen, Trans. Soc. Rheol. 5, 23 (1961).

${ }^{2}$ J. L. Eriksen, Arch. Ration. Mech. Anal. 9, 371 (1962).

${ }^{3}$ F. M. Leslie, Q. J. Mech. Appl. Math. 19, 357 (1966).

${ }^{4}$ O. Parodi, J. Phys. (Paris) 31, 581 (1970).

${ }^{5}$ G. Vertogen and W. H. de Jeu, Thermotropic Liquid Crystals, Fundamentals (Springer, Berlin, 1988).

${ }^{6}$ F. Z. Yang and J. R. Sambles, J. Opt. Soc. Am. B 16, 3 (1999).

${ }^{7}$ F. Yang, L. Ruan, and J. R. Sambles, J. Appl. Phys. 88, 11 (2000).

${ }^{8}$ S. A. Jewell and J. R. Sambles, J. Appl. Phys. 92, 1 (2002).

${ }^{9}$ N. J. Smith and J. R. Sambles, Appl. Phys. Lett. 77, 2632 (2001).

${ }^{10}$ L. Ruan and J. R. Sambles, J. Appl. Phys. 92, 9 (2002).

${ }^{11}$ D. Y. K. Ko and J. R. Sambles, J. Opt. Soc. Am. A 5, 1863 (1988). 